\title{
Working memory for color in honeybees
}

\author{
MICHAEL F. BROWN, DANIEL MCKEON, TIMO'THY CURLEY, \\ BRIAN WESTON, CONSTANCE LAMBERT, and BRIAN LEBOWITZ \\ Villanova University, Villanova, Pennsylvania
}

\begin{abstract}
Honeybees were tested in delayed conditional discrimination procedures (matching-to-sample and nonmatching-to-sample), using color stimuli presented on a video monitor. A small but reliable tendency to choose the color presented as the conditional cue was found, regardless of whether the contingencies reinforced or discouraged this tendency. The perseverative tendency occurred even with a delay of up to 1-2 min between the conditional cue and the choice. The tendency cannot be explained by changes in the associative value of the colors. Explanation of the results requires some form of working memory for color.
\end{abstract}

Many vertebrate species have been shown to exhibit the flexible, dynamic form of short-term memory that is often termed working memory. Working memory is typically thought of as holding discreet items of information, usually for relatively short periods of time (see, e.g., Honig, 1978). Two particularly well-studied examples are the memory for form or color stimuli exhibited by pigeons in the matching-to-sample paradigm (e.g., Roberts \& Grant, 1974 ) and the memory for spatial locations exhibited by rats in the radial-arm maze (Olton \& Samuelson, 1976).

Brown and Demas (1994; Demas \& Brown, 1995) recently presented evidence for spatial working memory in honeybees. Their procedure was designed to be an analogue of the rat radial-arm maze procedure. They reported a small but reliable tendency for bees to avoid revisits to locations recently depleted of food, just as rats display a very robust tendency to avoid revisits to locations recently depleted of food. Brown, Moore, Brown, and Langheld (1997) replicated and extended these results under a variety of experimental conditions. In contrast, Burmeister, Couvillon, and Bitterman (1995) failed to find evidence that spatial choices were controlled by the identity of previous spatial choices. More recently, Isnec, Couvillon, and Bitterman (1997) reported four experiments in which bees' spatial choices were controlled by the identity of previous choices. However, rather than the "winshift" (alternation) tendency found in our laboratory, this control was expressed as the opposite "win-stay" (perseverative) tendency in Isnec et al.'s subjects. Demas and Brown (1995) reported that bees are predisposed to alternate among reinforced spatial locations but could learn to perseverate under the appropriate reinforcement contingencies. In contrast, Isnec et al.'s subjects exhibited perseveration regardless of the contingencies. Although the

This research was supported by National Science Foundation Grant IBN-9404120. The participation of D.M. and T.C. was made possible by an NSF Research Experience for Undergraduates award. Correspondence should be directed to M. F. Brown, Department of Psychology, Villanova University, Villanova, PA, 19085 (e-mail: mbrown@email.vill.edu). explanation for the discrepancies in the manner in which previous visits affect subsequent choices is unclear, there is agreement that the results of these experiments require a memory system that allows discrimination of locations previously visited within a trial from those not yet visited (Bitterman, 1996; Brown et al., 1997; Isnec et al., 1997).

The existence of working memory in honeybees is of interest because of the vast differences between the structure of the honeybee's nervous system and those of the vertebrates in which working memory has been well studied (see, e.g., Chapman, 1982). To the extent that vertebrate and invertebrate working memory turn out to be similar in function, these similar functions must be implemented by very different neural structures. Because of this, a comparative analysis of working memory that includes invertebrates is likely to lead to important insights.

In the recent experiments demonstrating working memory in honeybees, spatial locations have served as the tobe-remembered stimulus. It is important to determine whether bee working memory can be used in other stimulus domains. In the present experiments, we were concerned with honeybee working memory for color. There is a wealth of evidence that honeybees can learn to respond differentially on the basis of color (see Bitterman, 1996; Gould, 1993, for recent reviews). Thus, some form(s) of long-term or reference memory must exist in bees that can store color information. Ohyama, Couvillon, and Bitterman (1995) recently provided the first evidence directly relevant to the possibility that color information is also stored in a working memory system in bees. They reported that honeybees' choice of color (yellow or blue) depended on the color most recently visited. Their procedure was similar to the well-known nonmatching-to-sample (or oddity-from-sample) procedure. During each trial, the bee first landed on (and fed on) a sample color. After depleting the small amount of sucrose solution available on the sample, the bee chose between two test colors, one identical to the sample color and the other the alternative color. Only the alternative color contained sugar. The bees were allowed to move to the correct (alternative) color after in- 
correct choices of the matching color. The color of the sample varied from one trial to the next. In opposition to the contingencies, Ohyama et al. found that bees demonstrated a small tendency to incorrectly choose the matching color. The perseveration of color choices is directly analogous to the perseveration of spatial choice discussed above (Isnec et al., 1997).

The results of Ohyama et al. (1995) are consistent with the view that bee choice behavior can be affected by working memory for color as well as working memory for spatial locations. However, as Ohyama et al. pointed out, their results are open to nonmemorial explanations, because the sample color was still present at the time that each choice was made. In effect, theirs was a simultaneous conditional discrimination procedure rather than a delayed conditional discrimination procedure. Thus, it did not require the persistence of information about sample identity across a temporal gap. In the present experiments, a delayed conditional discrimination procedure was used. The procedure was similar in many other respects to the one used by Ohyama et al. The experiments were intended to examine whether honeybees would be capable of making delayed conditional discriminations on the basis of color. The colors used in these experiments (green and violet) were within the range of hues that are highly discriminable for honeybees (see, e.g., Backhaus, 1992).

\section{EXPERIMENT 1}

The stimuli (and sugar) were presented on a horizontal video monitor screen. Bees in two experimental groups were exposed to two different contingencies: (1) a matching-to-sample contingency in which choice of the color matching the sample on each trial was reinforced, and (2) a nonmatching-to-sample contingency in which choice of the color different from the sample on each trial was reinforced.

\section{Method}

Subjects. The subjects were 24 honeybees (Apis mellifera) from a full-sized hive located inside a campus building, with a tunnel leading from the hive through a window to the outside. The hive was three floors above the laboratory where data were collected.

Apparatus. Two very similar apparatus were used. They each consisted of a color computer monitor (NEC, Model JC-1402HMA, and Zenith, Model ZCM1492-1), associated with IBM-386 microcomputers. Both monitors were driven by VGA-standard color adapters. Stimuli were formed in a $640 \times 350$ color pixel space on the $27 \times 20 \mathrm{~cm}$ monitor screens. Each monitor was placed with the screen facing up and the screen surface close to a laboratory window, at approximately the same height as the window ledge. The two apparatus were located near two windows on different sides of the building.

Transparent plastic petri dishes $(6.0 \mathrm{~cm}$ in diameter and $1.5 \mathrm{~cm}$ deep; Fisher Scientific No. 08-757-13A) were placed on the surface of the monitor screen; they contained sugar solution $(50 \% \mathrm{v} / \mathrm{v}$ refined granular sugar and tap water) or tap water. These dishes were rinsed and dried between uses.

Procedure. Bees were recruited by first netting them as they left the hive, and transporting them to the laboratory in small plastic vials. The vial was then opened and placed (open end down) on a petri dish, which had a large drop of sugar solution in it. The dish was placed on the monitor surface. The color of a large $(16-\mathrm{cm}-$ diameter) circle displayed on the monitor cycled every $3 \mathrm{sec}$ between green (VGA color standard No. 2) and violet (VGA color standard No. 5). Once a bee started feeding from the solution, the vial was removed. The bee typically fed to repletion and then flew out the laboratory window. Bees that did not feed from the drop were released or destroyed.

Some of the bees exposed to the recruitment procedure returned to the laboratory and fed on baited dishes placed on the monitor surface for this purpose. While such a bee was feeding, it was marked with a small spot of paint (Testor's model paint) for purposes of identification. Once a marked bee returned to the laboratory a second time, it was considered a subject in the experiment, and other bees were released and prevented from obtaining sugar solution from the apparatus. The experimental procedure described below began immediately and continued until the subject completed the experimental protocol or ceased returning to the laboratory.

Each bee successfully recruited was assigned to either the matching-to-sample (MTS) condition or to the nonmatching-tosample (NMTS) condition. To counterbalance for effects of differences between the two apparatus (or the two experimenters who conducted the experiment, each using one of the apparatus), half of the bees in each condition were tested using each of the two apparatus (and experimenters).

During the intertrial interval (ITI) that preceded each experimental trial, the experimenter placed a dish on the location where the sample stimulus was to appear. A small (approximately 5- $\mu \mathrm{l}$ ) drop of sugar solution was centered in the dish. The sample stimulus was either a violet or a green circle $(7.0 \mathrm{~cm}$ in diameter) which appeared directly under this dish. When the bee landed on the sample, the experimenter pressed a key that allowed the computer program to time this event. While the bee was feeding on the sample, the experimenter placed dishes on the two locations where the two test stimuli would later appear. One of these dishes contained a large (approximately 50- $\mu \mathrm{l}$ ) drop of sugar solution, and the other contained the same-sized drop of water. For bees in the MTS condition, the sugar solution was in the location to be occupied by the test stimulus that was identical to the sample in color. For bees in the NMTS condition, the sugar solution was in the location to be occupied by the test stimulus that was different from the sample in color. A key was pressed when the bee left the dish in the sample location. This keypress recorded the time of this event. It also resulted in the sample stimulus' being removed from the screen. The dish remained in the sample location until the following ITI.

Immediately following removal of the sample stimulus from the monitor screen, the two test stimuli appeared under the dishes that had been placed in their locations. The test stimuli were $7.0 \mathrm{~cm}$ in diameter, separated by $10 \mathrm{~cm}$ (center-to-center), and were each $12.5 \mathrm{~cm}$ from the location where the sample stimulus had been presented (center-to-center). The test stimuli were aligned parallel to the window, with the sample location closer to the window (thus, the three stimulus locations formed a triangle, with the sample stimulus location closest to the window). When any part of the bee touched one of the dishes at a test location, that was defined as the test choice (and a key was pressed to indicate the identity and time of the choice). If the bee's choice was correct, it was allowed to feed on the sugar solution in the chosen location. If the bee's choice was incorrect, the dish on the correct test stimulus was removed so that the bee could not fly from the incorrect location to the correct location and receive sugar solution. A key was pressed when the bee left the dish in the chosen location. This removed both test stimuli from the monitor screen and defined the beginning of the ITI. Bees fed to repletion following a correct choice and then flew out the window (presumably to the hive, where they deposited the sugar solution). Typically, bees remained in the laboratory for a subsequent trial following an incorrect choice. There was a blank interval of 


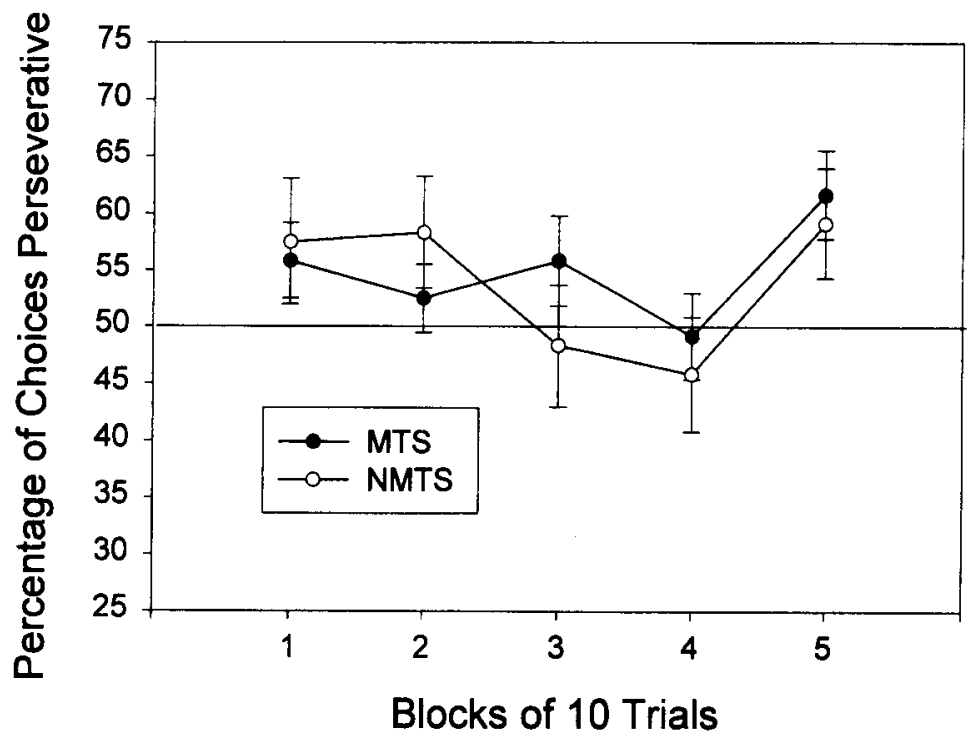

Figure 1. Mean percentage of trials with a perseverative choice of the test stimulus identical in color to the sample stimulus in the matching-to-sample (MTS) and nonmatching-to-sample (NMTS) groups over the course of trial blocks in Experiment 1. Perseverative choices were reinforced in the MTS group, but not in the NMTS group. Error bars represent standard error of the mean.

$10 \mathrm{sec}$ before the sample appeared for the next trial, after which the dish containing the small drop of solution was placed on the sample. The sample remained on the screen during the remainder of the ITI (i.e., until the bee landed on the sample location).

The identity of the sample (green or violet) and the location of the correct test stimulus (left or right) were randomized over trials. Each bee was tested for 50 trials.

\section{Results and Discussion}

The mean choice accuracy for the MTS group $(55.0 \%$ correct) was significantly greater than chance $[50 \%$; $t(11)=4.28$ ]. The mean choice accuracy for the NMTS group ( $46.2 \%$ correct) was significantly less than chance $[t(11)=2.26]$. To allow comparison of the behavior of bees in the two groups, performance in the NMTS group was converted from percentage correct to the (reciprocal) percentage of choices which were perseverative (i.e., incorrect choices of the test stimulus that was identical in color to the sample stimulus). Perseverative choices in the MTS group, of course, were correct. Figure 1 shows the data in these terms across the five trial blocks. An analysis of variance (ANOVA) failed to reveal any evidence that choice tendency varied as a function of group $[F(1,22)<1]$ or across trial blocks $[F(4,88)=2.17]$. Nor was there any evidence that the effects of these variables interacted $[F(4,88)<1]$.

Honeybees under these experimental conditions demonstrated a small but reliable tendency to choose the color that matched the sample, regardless of whether the contingencies were designed to encourage matching or nonmatching. In fact, there was no evidence in these choice accuracy data that the reinforcement contingencies affected behavior. This is consistent with the results of Isnec et al. (1997), who found perseveration in the spa- tial domain regardless of whether the contingencies encouraged matching, nonmatching, or neither. On the other hand, this result is in opposition to the results found in our laboratory when bees respond on the basis of spatial location (Brown \& Demas, 1994; Brown et al., 1997; Demas \& Brown, 1995). Discussion of possible explanations for these apparent contradictions will be deferred to the General Discussion. Regardless of the behavioral tendencies of the bees in response to the sample color, the fact that choices were affected by the identity of the previously presented sample color means that working memory for color exists in bees.

Figure 2 provides means of the durations of three trial events over the course of trial blocks: the ITI, the time spent on the sample (sample time), and the time between leaving the sample and choosing a test stimulus (choice time). These event durations are shown as a function of trial block, experimental group, and the outcome of the trial (correct vs. incorrect; in the case of ITI, the data are coded in terms of the outcome of the preceding trial). A series of three analogous ANOVAs (group [MTS vs. NMTS] $\times$ trial block $\times$ outcome of choice) was used to interpret these results. ITIs were longer following correct trials than following incorrect trials $[F(1,22)=69.4]$. This can be attributed to the fact that bees fed to repletion and left the laboratory following a correct choice, but typically remained in the laboratory during the ITI following an incorrect choice. ITIs were also longer in the NMTS group than in the MTS group $[F(1,22)=4.4]$. There was no effect of trial block, nor did the effects of these factors interact. Sample times decreased over the course of trial blocks $[F(4,88)=5.2]$ but did not differ as a function of any of the other factors. Choice times were longer 


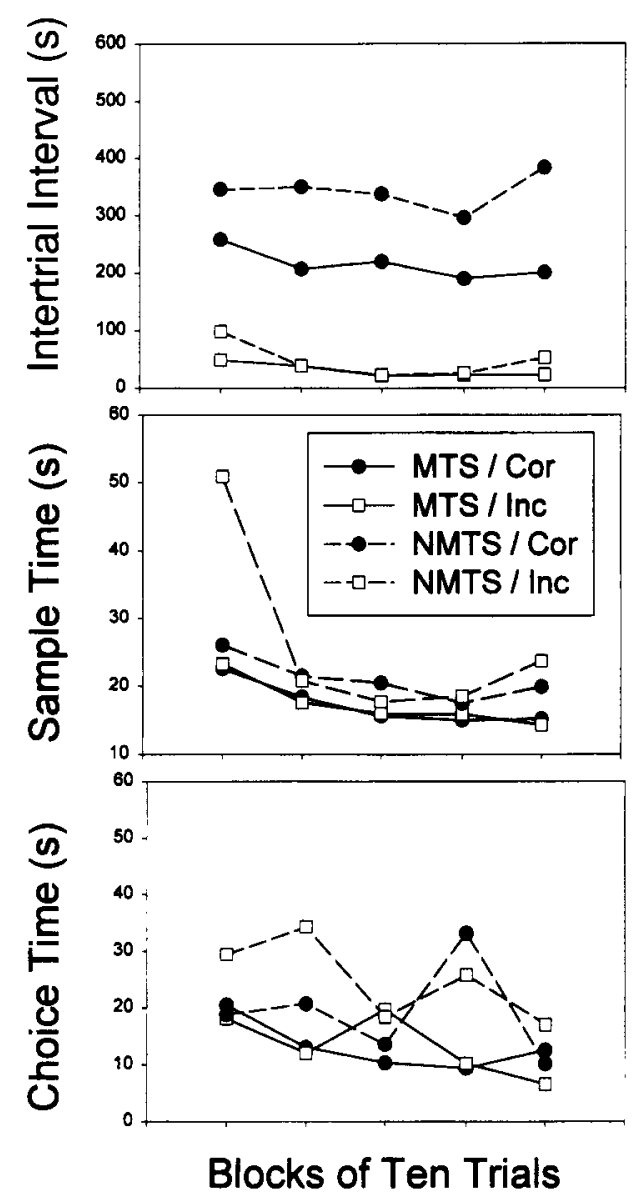

Figure 2. Mean values of selected temporal intervals as a function of experimental group and trial outcome over the course of the trial blocks of Experiment 1. MTS, matching-to-sample; NMTS, nonmatching-to-sample; Cor, correct; Inc, incorrect. Trial outcome refers to the previous trial in the case of intertrial interval and the current trial in the case of sample time and choice time.

in the NMTS group than in the MTS group $[F(1,22)=$ 6.4] but did not differ as a function of any of the other factors. One possible interpretation of the group difference is that it was a subtle effect of the conflict between the perseverative tendency and the NMTS contingencies.

\section{EXPERIMENT 2}

The results of Experiment 1 demonstrate control of choice by a previously presented color, and therefore indicate the existence of working memory for color in honeybees. However, the magnitude of this control is small, just as the magnitude of spatial working memory in bees has been found to be small (Brown \& Demas, 1994; Brown et al., 1997; Isnec et al., 1997). It is of interest to examine whether there is a fundamental limitation on the degree of working memory ability in bees or whether the limits on working memory abilities found thus far were a matter of the specific experimental procedures used in the extant experiments.

In Experiment 2, we examined the effect of a delay interval (imposed between exposure to the sample stimulus and the test choice) on choice accuracy. Although mean choice times in Experiment 1 were on the order of $10-25 \mathrm{sec}$, our observations of bee behavior during the choice interval suggested that bees did not always observe both test stimuli prior to making a choice, and therefore may have made some choices that were not under stimulus control. In Experiment 2, a clear plastic barrier was placed between the sample stimulus location and the test stimuli to force bees to fly high enough so that both test stimuli would be clearly visible prior to making the choice. We expected this procedure to increase the extent to which test choices were under stimulus control. Of course, a tall barrier might also be expected to impose a long enough delay between sample stimulus presentation and test stimulus choice so that memory performance would be detrimentally affected.

Three experimental conditions were used: no barrier, a short barrier, and a tall barrier. The short barrier was intended to increase stimulus control of choices, while imposing only a small increase in the interval from sample presentation to choice response (choice time). Thus, it was expected that the short barrier might result in increased levels of choice accuracy, relative to the no-barrier control condition. On the other hand, the tall barrier was expected to impose a relatively large increase in choice time, thereby decreasing choice accuracy relative to the nobarrier condition.

\section{Method}

Subjects. The subjects were 19 honeybees from the same source as those used in Experiment 1.

Apparatus. The apparatus were the same as those used in Experiment 1 , except for the addition of enclosure and barrier systems on top of each video monitor. The enclosure was constructed of two plywood panels, held together with four struts. The two panels ( $35 \mathrm{~cm}$ tall $\times 20 \mathrm{~cm}$; painted flat black) were aligned with the two sides of the monitor screen, such that the bees were contained within the space defined by the sides of the monitor screen surface. The front and back of the enclosure (facing the window and the experimenter) were open (except for the small struts attached to the panels), as was the top. Tracks mounted on the inside surface of the panels allowed clear Plexiglas barriers to be mounted in a vertical plane dividing the area of the screen in which the sample stimulus was presented from the area of the screen in which the test stimuli were presented. The barriers were $24 \mathrm{~cm}$ long, spanning the width of the enclosure. Two barrier heights were used in the experiment: $6 \mathrm{~cm}$ tall and $30 \mathrm{~cm}$ tall. The barriers could be easily removed and/or replaced during the ITI. The enclosure and barrier system required the bees to move from the sample location to the test stimuli's locations by flying over the barrier, if one was present.

Procedure. Ten bees were tested in one of the two apparatus used in Experiment 1, and the remaining 9 bees were tested in the other apparatus. The testing procedure was identical to that used in MTS group of Experiment 1, with the following exceptions: The enclosure was present throughout each trial. Trials were run in blocks of 3 each, with each of the three barrier conditions (no barrier, short barrier, tall barrier) being in force during 1 trial (in a randomized order). Each bee was tested for 60 trials. 


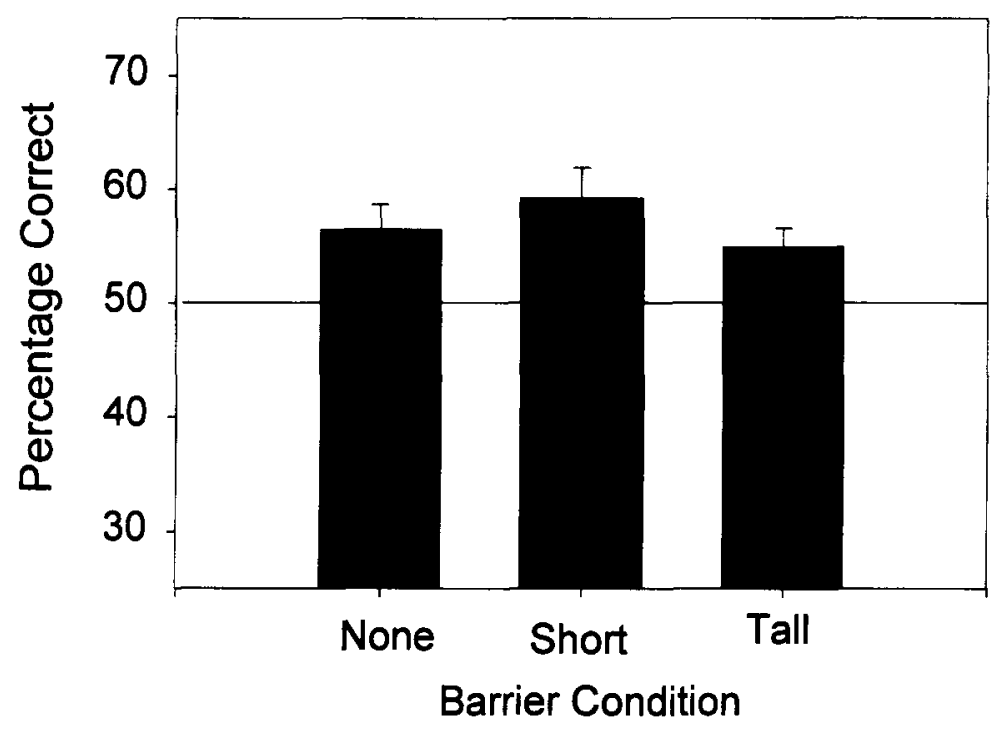

Figure 3. Mean choice accuracy in Experiment 2 as a function of barrier condition. Error bars represent standard error of the mean.

\section{Results and Discussion}

Figure 3 shows the mean choice accuracy of the bees as a function of barrier condition. Mean choice accuracy collapsed across barrier condition $(56.8 \%)$ was greater than chance $[50 \% ; t(18)=6.27, p<.001]$. However, there was no evidence that choice accuracy varied as a function of barrier condition $[F(2,36)<1]$. As expected, the use of barriers did increase the interval between exposure to the sample and choice of a test stimulus. Mean choice times for the no-barrier, short barrier, and tall barrier conditions were $19.3,42.2$, and $91.3 \mathrm{sec}$, respectively. These values are reliably different $[F(2,36)=12.9, p<.001]$.

The present results provide no evidence for the possibility that a lack of stimulus control by test stimuli limits working memory performance or for an effect of retention interval on working memory performance in bees. However, the results do replicate the basic result of Experiment 1 that bees choose a color stimulus on the basis of the identity of a recently presented color.

\section{EXPERIMENT 3}

One potential factor in the perseverative tendency of bees to choose the test color that matches the sample is the fact that sugar solution is available (and always imbibed) on the sample stimulus. The receipt of sugar on the sample might be expected to increase the associative value of the sample color. If the relative associative strength of the two colors can sufficiently fluctuate on a trial-by-trial basis, such changes might account for the perseveration found in the first two experiments.

An associative interpretation cannot explain the tendency of bees to alternate ("win-shift") when sugar is received in a spatial location (Brown \& Demas, 1994;
Brown et al., 1997; Demas \& Brown, 1995), because such an associative effect would produce the opposite perseverative tendency. Isnec et al. (1997), who found a perseverative tendency for visits to spatial locations, considered the possibility that it was due to changes in the associative value of the spatial location. They rejected that idea, however, on the basis of their failure to find any evidence of cumulative effects of reinforcement over visits. That is, the perseverative tendency of their bees was no greater when sugar had been found on the color for several choices in succession than when sugar had been found on the color only on the last visit. An associative process would be expected to produce cumulative effects of reinforcement.

In the present experiment, we examined the possibility that the sugar solution provided on the sample color during each trial of Experiments 1 and 2 was responsible for the perseverative tendency found in those experiments. Rather than look for cumulative effects of reinforcement over trials, we manipulated (over trials) whether or not sugar solution was provided on the sample color. If the provision of sugar on the sample was responsible for the results of the previous experiments, a perseverative tendency should be obtained only on the trials that included sugar on the sample.

\section{Method}

Subjects and Apparatus. The subjects were 10 honeybees from the same source as that of those used in Experiment 1. The apparatus was the same as one of those used in Experiment 1 (the one that included the Zenith monitor).

Procedure. The testing procedure was identical to that used in the MTS group of Experiment 1, with the following exceptions: The size of the sugar solution or water drop placed on the test stimuli was the same as that placed on the sample stimulus (approximately 


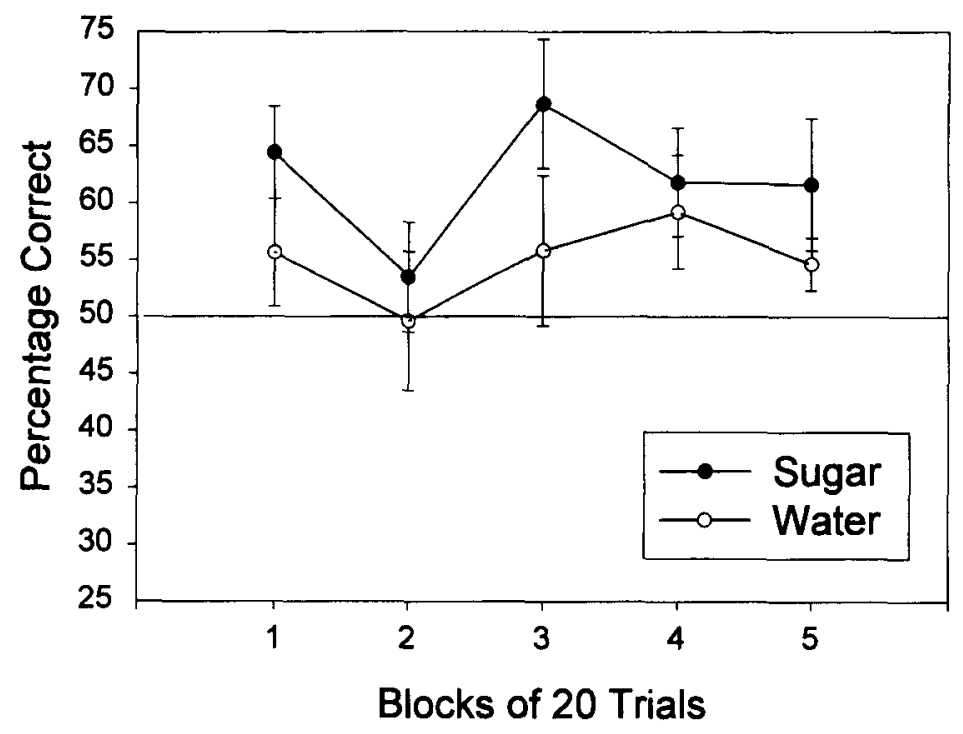

Figure 4. Mean choice accuracy of the bees in Experiment 3 , as a function of trial block and sample solution condition. Error bars represent standard error of the mean.

$5 \mu \mathrm{l}$ ). This change was introduced to increase the size (and possibly the effect) of the sugar on the sample stimulus relative to the sugar on the correct test stimulus. As a result, the bees did not always leave the laboratory following a correct choice (as they did in Experiments 1 and 2). The bees were tested for 100 trials. On a randomly selected half of these trials, the dish placed on the sample stimulus contained water. On the remaining half of the trials, the dish contained sugar solution.

\section{Results and Discussion}

Nine bees completed the scheduled protocol of 100 trials. A 10 th bee completed 60 trials, after which it ceased returning to the laboratory for unknown reasons.

Mean choice accuracy (over the 100 trials for 9 bees and 60 trials for the 10th) was greater than that expected by chance $(50 \%)$ both when there was sugar solution on the sample $[61.9 \% ; t(9)=4.29, p<.01]$ and when there was water on the sample $[54.6 \% ; t(9)=3.21, p<.01]$. There was also a significant difference between choice accuracy with water and sugar on the sample $[t(9)=2.39, p<.05]$.

To examine the possibility of changes in the pattern of results over the course of the experiment, the data were divided into trial blocks of 20 trials each and examined in an ANOVA. Figure 4 shows the mean percentage of trials with a correct choice, over five blocks of 10 trials each, for trials with a water drop or a sugar drop on the sample. There was no evidence for an effect of block $[F(4,32)=1.65]$ or for an interaction between effects of block and the substance on the sample $[F(4,32)<1]$. In conflict with the results of the $t$ test, the ANOVA provided no evidence that choice accuracy was greater when sugar was provided on the sample $[F(1,8)=3.59, p=.09]$.

The critical result for purposes of this experiment is that perseveration occurred whether or not sugar was provided on the sample stimulus. Thus, the perseverative tendency of bees in these experiments cannot be explained solely as an associative effect. This finding strongly sup- ports the interpretation of the tendency of bees to choose the matching color as depending on working memory for the identity of the most recently presented color.

On the other hand, it may be that an associative effect did increase the extent to which perseveration occurred in the present experiment, as it appears to have done in other experimental procedures (e.g., Greggers \& Mauelshagen, 1997; Greggers \& Menzel, 1993; Grossmann, 1973). However, this possibility should be applied to the present results with caution, for two reasons. First, the statistical analyses of the present results provide only equivocal evidence that choice accuracy was higher in the sugar sample condition than in the water sample condition. Second, even if such an effect did occur in the present experiment, it can be explained either as an associative effect or as a working memory effect. The latter explanation follows from the fact that bees spent more time on the sample when there was sugar solution (mean over bees $=28.0 \mathrm{sec}$ ) than when there was water [mean $=5.0 \mathrm{sec}$; the difference is significant, $t(9)=8.5, p<.001]$. It is well known that the duration of a color sample strongly affects the accuracy of matching-to-sample performance in pigeons and other animals (see, e.g., Roberts \& Grant, 1974). Thus, it is possible to attribute an effect of sample solution in the present experiment to a confounded effect of sample duration on working memory performance. In any event, even if an associative effect modulated the degree of perseveration, perseveration occurred regardless of reinforcement and therefore must be due at least in part to a nonassociative process.

\section{GENERAL DISCUSSION}

These results show that bees' choice of a color stimulus from two possibilities is influenced by the identity of the most recently visited color. The interval between the 
sample color and the choice can be as long as 1-2 min. The attraction to the matching test color cannot be explained in terms of changes in the relative associative strength of the colors, because it occurs whether or not the sample stimulus has been reinforced (see also Isnec et al., 1997). Thus, the present results are best interpreted as resulting from a memory for the identity of the most recently visited color. The use of computer-controlled stimuli allowed us to be certain that sample information was not physically present at the time when the subject chose a test stimulus. Thus, the control of bees' choice of test stimuli by the identity of the sample stimulus corresponds to the essential features of vertebrate working memory performance (Baddeley, 1986; Honig, 1978). The identity of the sample color changes unpredictably from trial to trial, and the relative attractiveness of the color test stimuli changes as a function of sample identity. Thus, the nervous system of bees flexibly and temporarily stores the identity of the most recently presented sample. We therefore conclude that these data provide evidence for working memory for color in bees.

Bee working memory ability is not limited to spatial location (Brown \& Demas, 1994; Brown et al., 1997; Isnec et al., 1997), but may instead be a general purpose system, as it appears to be in vertebrates. The level of working memory performance demonstrated in these experiments was low, in agreement with the levels of honeybee working memory performance found in experiments using spatial locations as the to-be-remembered stimuli (Brown \& Demas, 1994; Brown et al., 1997; Isnec et al., 1997). This is consistent with the possibility that performance in the two cases is limited by the properties of a common memory system.

Memory for the identity of a recently visited stimulus could affect behavior in either of two ways: The tendency of the bee to visit that stimulus might either increase (perseveration) or decrease (alternation). As discussed above, conflicting results have been reported from delayed conditional discriminations involving spatial location. In our laboratory, an alternation tendency has been repeatedly found (Brown \& Demas, 1994; Brown et al., 1997; Demas $\&$ Brown, 1995), whereas Isnec et al. (1997) have reported a perseveration tendency. The explanation of this discrepancy in the results regarding working memory performance in the spatial domain remains unclear (Brown et al., 1997). The present results show a perseverative tendency when color is the conditional cue, in agreement with the results of Ohyama et al. (1995).

Perseveration to color cues and alternation to spatial locations can be interpreted in the context of bee natural history. It is well known that honeybees tend to forage on one particular species of flower at a time ("flower constancy"; see, e.g., Free, 1963; Hill, Wells, \& Wells, 1997) and color is thought to be one of the primary cues that bees use to discriminate among flower species (e.g., Winston, 1987). Thus, perseveration to colors may be related to flower constancy (Ohyama et al., 1995). On the other hand, it would be to bees' advantage to avoid revisits to specific flowers, because those will generally be depleted of nectar for a substantial interval following a visit (Brown \& Demas, 1994). Thus, because specific flowers correspond to spatial locations, alternation in the spatial domain also makes ecological sense. These tendencies to alternate spatial locations and perseverate on colors may or may not involve learning. In laboratory experiments, the tendency to perseverate on a particular color has shown little or no change with experience (Ohyama et al., 1995; present experiments), suggesting that the tendency to return to a particular color is not produced by a learning process. The role of experience in producing an alternation tendency in the spatial domain is less clear, given the discrepancies reviewed above. However, there is at least some evidence of flexibility in the tendency of bees to avoid revisits to locations where food has been found (Demas \& Brown, 1995), suggesting that this tendency may be sensitive to its consequences.

Whatever the details, it is clear that information about recent visits can affect subsequent choices of honeybees, thereby implicating working memory ability. The present experiments show that this ability is not restricted to the spatial domain, and that it can also be applied to color. Among the important remaining questions are the relationship between the working memory used for spatial location and that used for color, as well as the relation between bee working memory and that studied in vertebrate animals.

\section{REFERENCES}

Backhaus, W. (1992). Color vision in honeybees. Neuroscience \& Biobehavioral Reviews, 16, 1-12.

BaddelEy, A. D. (1986). Working memory. Oxford: Oxford University Press.

BitTERMan, M. E. (1996). Comparative analysis of learning in honeybees. Animal Learning \& Behavior, 24, 123-141.

Brown, M. F., \& DEMAs, G. E. (1994). Evidence for spatial working memory in honeybees (Apis mellifera). Journal of Comparative Psychology, 108, 344-352.

Brown, M. F., Moore, J. A., Brown, C. H., \& Langheld, K. D. (1997). The existence and extent of spatial working memory ability in honeybees. Animal Learning \& Behavior, 25, 473-484.

Burmeister, S., Couvillon, P. A., \& Bitterman, M. E. (1995). Performance of honeybees in analogues of the rodent radial maze. Animal Learning \& Behavior, 23, 369-375.

ChAPMAN, R. F. (1982). The insects: Structure and function (3rd ed.). Cambridge, MA: Harvard University Press.

Demas, G. E., \& Brown, M. F. (1995). Honeybees are predisposed to win-shift but can learn to win-stay. Animal Behaviour, 50, 104 1-1045.

FrEe, J. B. (1963). The flower constancy of honeybees. Journal of Chemical Ecology, 32, 119-131.

GouLD, J. L. (1993). Ethological and comparative perspectives on honey bee learning. In D. R. Papaj \& A. C. Lewis (Eds.), Insect learning: Ecological and evolutionary perspectives (pp. 18-50). New York: Chapman \& Hall.

Greggers, U., \& MauelshaGen, J. (1997). Matching behavior of honeybees in a multiple-choice situation: The differential effect of environmental stimuli on the choice process. Animal Learning \& Behavior, $25,458-472$.

Greggers, U., \& Menzel, R. (1993). Memory dynamics and foraging strategies of honeybees. Behavioral Ecology \& Sociobiology, 32, 17-29. 
Grossmann, K. E. (1973). Continuous, fixed-ratio, and fixed-interval reinforcement in honeybees. Journal of the Experimental Analysis of Behavior, 20, 105-109.

Hill, P. S. M., Wells, P. H., \& Wells, H. (1997). Spontaneous flower constancy and learning in honey bees as a function of color. Animal Behaviour, 54, 615-627.

HoNIG, W. K. (1978). Studies of working memory in the pigeon. In S. H. Hulse, W. K. Honig, \& H. Fowler (Eds.), Cognitive processes in animal behavior (pp. 211-248). Hillsdale, NJ: Erlbaum.

Isnec, M. R., Couvillon, P. A., \& Bitterman, M. E. (1997). Shortterm spatial memory in honeybees. Animal Learning \& Behavior, 25 , 165-170.

Ohyama, T., Couvillon, P. A., \& Bitterman, M. E. (1995). Persever- ation in the color choices of honeybees. Journal of Insect Behavior, $\mathbf{8}$, 409-415.

Olton, D. S., \& SAmuelson, R. J. (1976). Remembrance of places passed: Spatial memory in rats. Journal of Experimental Psychology: Animal Behavior Processes, 2, 97-116.

Roberts, W. A., \& Grant, D. S. (1974). Short-term memory in the pigeon with presentation time precisely controlled. Learning \& Motivation, 5, 393-408.

Winston, M. L. (1987). The biology of the honey bee. Cambridge, MA: Harvard University Press.

(Manuscript received January 8, 1998; revision accepted for publication April 4, 1998.) 